\title{
Schlusswort und Ausblick
}

\author{
Andreas Mahr und Marcel Sailer
}

Liebe Leser*innen,

wir hoffen, dass unser hier vorliegender Überblick über den aktuellen Stand zum Thema Active Assisted Living (AAL) bei Ihnen mit dem ein oder anderen Vorurteil gegenüber „der Technikzentrierung“ aufgeräumt hat, Verständnis für den Nutzen und die Notwendigkeit der Entwicklungen geweckt und ganz prinzipiell Ihr Interesse am Thema geweckt oder vertieft hat.

Für uns als Hochschule hat sich das Thema AAL als Forschungsschwerpunkt über alle Fakultäten hinweg entwickelt. Wie oft bei neuen vermeintlich technikzentrierten Themen, haben sich anfänglich vor allem die technischen Disziplinen der Informatik und der Ingenieurswissenschaften des Themas angenommen. Sehr schnell wurde allen aber bewusst, dass die eigentliche Herausforderung des Themas in der interdisziplinären Zusammenarbeit aller Fakultäten der Hochschule liegt.

Der derzeitige Stand der Verbreitung von AAL-Produkten zeigt, dass sich zukünftige Forschung und Entwicklung des Gebietes eher auf den Nutzen und die Bereitschaft zur Anwendung der Zielgruppe orientieren sollte. Es fehlt nicht an Produkten, sondern an einer wissenschaftlichen Aufarbeitung des Nutzens und der eigentlichen Bedürfnisse der Zielgruppen der Produkte. Hier kann eine interdisziplinäre Zusammenarbeit von Informatik, Ingenieurswissenschaften, Gesundheitsund Sozialwissenschaften einen echten Mehrwert generieren und die Thematik aus völlig neuen Blickwinkeln betrachten.

\footnotetext{
A. Mahr $(\bowtie) \cdot$ M. Sailer

DHBW Heidenheim, Heidenheim, Deutschland

E-Mail: andreas.mahr@dhbw-heidenheim.de

M. Sailer

E-Mail: marcel.sailer@dhbw-heidenheim.de
} 
Im ganzen Land eröffneten nun Reallabore, um sich der Thematik technischer Assistenzsysteme anzunehmen. Konzentriert sind diese Reallabore jedoch meist auf den städtischen Raum. Gerade jedoch im ländlichen Bereich zeigen sich bereits heute erhebliche Versorgungslücken in der Gesundheitsversorgung. Insbesondere dort, wo schon jetzt ein Ärzt*innen und Pflegepersonalmangel herrscht, Apotheken und Sanitätshäuser geschlossen wurden, Therapeuten nur durch weite Anfahrten zu erreichen - und vorhanden Dienste stark überlastet sind. Hier müssen schnell wegweisende Einrichtungen und Konzepte weiterentwickelt werden, um die Herausforderungen durch neue, innovative Ansätze zusätzlich zu unterstützen. Darin spiegelt sich auch die grundlegende Philosophie in diesem Werk, welches die Lösung eben nicht in einer simplen technischen Kompensation des Personalmangels sieht. Auch wenn die schiere Not oder ökonomische Beweggründe solche Ansätze befeuern. Letzten Endes werden sich nur die Systeme durchsetzen, welche komplementär unterstützen, die Autonomie und Selbstständigkeit der Personen fördern, und Sozialkontakte und menschliche Interaktion nicht reduzieren, sondern im Gegenteil fördern.

Hier kann die Duale Hochschule unterstützen, initiieren und fördern. Gegründet in den 70er Jahren, um wissenschaftliche Einrichtungen auch in Regionen zu bringen, in denen keine klassischen Universitäten angesiedelt sind und in denen dennoch ein hoher Bedarf der Wirtschaft und sozialen Einrichtungen an wissenschaftlich ausgebildeten Fachkräften besteht, spielt die DHBW nach über 40 Jahren Ihrer Gründung nicht nur eine wichtige Rolle in der Fachkräftesicherung, sondern ist sich zunehmend auch Ihrer gesellschaftlichen Verantwortung bewusst. Im Rahmen Ihrer „Third Mission“ rückt die Hochschule mit dem hier behandelten Thema AAL mitten in eines der gesellschaftlich herausforderndsten Themen der kommenden Jahre und Jahrzehnte. Der Anstoß für die Publikation wurde durch die interprofessionelle Arbeit der Studierenden der Gesundheits- und Pflegewissenschaften und Wirtschaftsinformatik gegeben. Bereits im ersten Aufeinandertreffen wurde deutlich, welch innovative Ideen sich entwickeln, wenn der "Think Tank" im Seminar eröffnet wurde. Während im ersten Durchlauf der Themenbereich AAL hinsichtlich deren Potenziale und Anwendungsgebiete grundsätzlich erörtert wurde, so beschäftigen sich die Folgejahrgänge nun mit konkretisierten Problemstellungen, um den Anwendungsbezug weiter $\mathrm{zu}$ fokussieren. Im laufenden Studienjahr beschäftigen sich Studierende mit dem Thema AAL bei dementiellen Erkrankungen. Entsprechend der Entwicklungen der Hochbetagten als Hauptzielgruppe für technologische Assistenzsysteme, und dem einhergehenden Risiko beziehungsweise bestehendem Anstieg an dementiellen Erkrankungen in Deutschland entsteht ein spezifischer, komplexer Bedarf für die Hilfesysteme. Schließen sich die technologischen Hilfen bei kognitiven Einschränkungen 
dadurch aus, oder benötigen Sie gerade deshalb adaptierte Konzepte? Im Kontext dieser interessanten Herausforderungen beschäftigen sich Studierende mit einer digital unterstützten Biographiearbeit, einem digitalen Medikamentendispenser, der digital unterstützten Trinküberwachung und - stimulation, mit Smart Wearables, dem Managed Food Service sowie dem Einsatz der Robotik.

Aktuell wird entsprechend der Konzeption von Dr. Reichstein (Abschn. 7.4) begleitend die Einrichtung eines Reallabors in Heidenheim eingeleitet. In diesem sollen die innovativen Konzepte der Studierenden gemeinsam mit der Bevölkerung sowie mit Herstellern und Dienstleistern diskutiert und weiterentwickelt werden. Im Vordergrund stehen hier die Interaktion und Kommunikation mit den Nutzer*innen, um die im Buch präferierte nutzerorientierte Konstruktion und Entwicklung zu fördern. Idealerweise wird sich dann ein AAL-Musterhaus als feste Anlaufstelle für Fachpublikum und breite Öffentlichkeit in der Region Ostwürttemberg etablieren, um Hemmschwellen abzubauen, Neues begreif- und erfahrbar zu machen und die anwendungsbezogene Forschung auf dem Gebiet voran zu treiben.

Dieses Buch soll einen Grundstein für die Verankerung der Thematik im Aufgabenbereich der Hochschule legen. Wir bedanken uns abschließend noch einmal sehr herzlich für die anregende und sehr angenehme Zusammenarbeit bei allen mitwirkenden Autor*innen. Ein besonderer Dank gilt außerdem Frau Dr. Petra Weber und Herrn Dr. Christopher Reichstein für die redaktionelle und wissenschaftliche Unterstützung.

Durch die Mitwirkung kreativer und engagierter Studierender sowie Hochschuldozent*innen aller Fachrichtungen wurde damit ein erster Schritt getan, viele weitere müssen folgen. Packen wir es an!

Andreas Mahr und Marcel Sailer

Im März 2021 

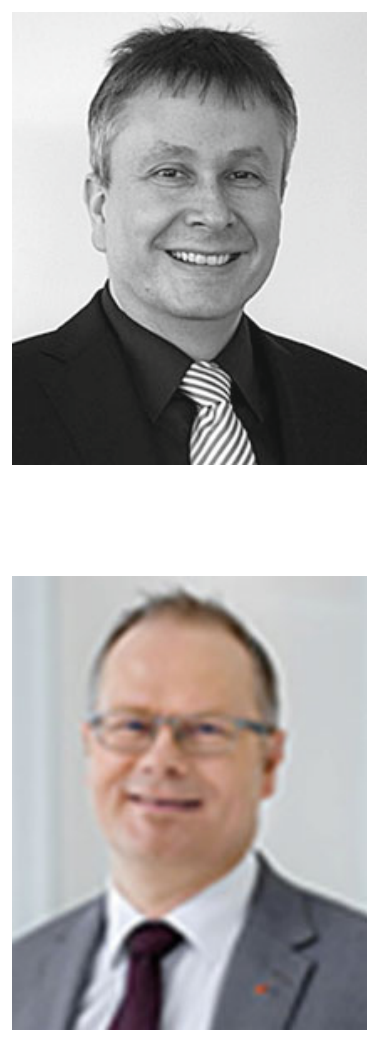

Andreas Mahr Prorektor Forschung und Dekan der Fakultäten Technik und Gesundheit an der DHBW Heidenheim.

Seit 20 Jahren als Professor an der Hochschule und zuvor am Deutschen Krebsforsungszentrum in Heidelberg tätig. Neben Schwerpunkten in der Informatik befasste sich der promovierte Medizin-Informatiker in den vergangenen 10 Jahren intensiv mit dem Umbruch der Ausbildung und der zunehmenden Digitalisierung im Gesundheitsbereich. Als langjähriger Studiengangsleiter in der Medizinischen Informatik betreute er Kliniken in ganz Süddeutschland und erhielt einen vertieften Einblick in die wachsende Rolle und Bedeutung der Informationstechnologien in Krankenhaus und Gesellschaft. Im Rahmen der Third Mission der Hochschule liegt dem Autor besonders das Thema digitalisierte Gesellschaft am Herzen.

Marcel Sailer Prodekan Gesundheit, Studiengangsleiter für Angewandte Gesundheits- und Pflegewissenschaften an der Dualen Hochschule Baden-Württemberg Heidenheim.

Seit mehr als 30 Jahren ist er als Krankenpfleger, Pflegepädagoge und Humanbiologe in verschiedenen ambulanten und stationären Einrichtungen des Gesundheitssystems tätig. Seit 2013 belegt er den Lehrstuhl für Gesundheitsund Pflegewissenschaften. Seine Schwerpunktthemen konzentrieren sich auf onkologische und geriatrische Versorgungssysteme, Pflegediagnostik und klinische Entscheidungsfindung, klinisches Prozessmanagement, Patientenedukation, Personalentwicklung und betriebliche Bildung, klinische Ökonomik und Blended learning-Szenarien. Er arbeitet als Berater für Gesundheits- und Bildungseinrichtungen und Coach für Führungskräfte. Als Gründungsmitglied im Netzwerk Gesundheit \& Pflege Heidenheim beschäftigt er sich seit mehreren Jahren mit der Versorgung älterer Menschen in ländlichen Regionen. 
Open Access Dieses Kapitel wird unter der Creative Commons Namensnennung 4.0 International Lizenz (http://creativecommons.org/licenses/by/4.0/deed.de) veröffentlicht, welche die Nutzung, Vervielfältigung, Bearbeitung, Verbreitung und Wiedergabe in jeglichem Medium und Format erlaubt, sofern Sie den/die ursprünglichen Autor(en) und die Quelle ordnungsgemäß nennen, einen Link zur Creative Commons Lizenz beifügen und angeben, ob Änderungen vorgenommen wurden.

Die in diesem Kapitel enthaltenen Bilder und sonstiges Drittmaterial unterliegen ebenfalls der genannten Creative Commons Lizenz, sofern sich aus der Abbildungslegende nichts anderes ergibt. Sofern das betreffende Material nicht unter der genannten Creative Commons Lizenz steht und die betreffende Handlung nicht nach gesetzlichen Vorschriften erlaubt ist, ist für die oben aufgeführten Weiterverwendungen des Materials die Einwilligung des jeweiligen Rechteinhabers einzuholen. 